\title{
A Novel Gas Sensor from Poly(ethylene glycol)-Grafted Carbon Black. Responsibility of Electric Resistance of Poly(ethylene glycol)- Grafted Carbon Black against Humidity and Solvent Vapor
}

\author{
Masaki OKaZaKi, Kiyotaka MaruYama, Masaki Tsuchida, ${ }^{*}$ \\ and Norio TsuboKawa ${ }^{*} \dagger$ \\ Graduate School of Science and Technology, Niigata University, \\ 8050 Ikarashi 2-nocho, Niigata 950-2181, Japan \\ * Department of Material Science and Technology, Faculty of Engineering, \\ Niigata University, 8050 Ikarashi 2-nocho, Niigata 950-2181, Japan
}

(Received February 16, 1999)

\begin{abstract}
Poly(ethylene glycol) (PEG) having different molecular weight was successfully grafted onto carbon black surface by direct condensation of terminal hydroxyl groups of PEG with carboxyl groups on the surface using $N, N^{\prime}$ dicyclohexylcarbodiimide as a condensing agent. The electric resistance of a composite prepared from PEG and PEGgrafted carbon black drastically increased to $10^{3}-10^{4}$ times of initial resistance in humidity and vapor of methanol, and ethanol, which are good solvent of PEG, and returned immediately to initial resistance when it was transferred in dry air. But the change of electric resistance of the composite was hardly observed in hexane and toluene vapor, which are poor solvent of PEG. The logarithm of electric resistance is linearly proportional to relative humidity. The sensitivity of electric resistance decreased with increasing molecular weight of PEG. In addition, the electric resistance of the composite from PEG-grafted carbon black lost the responsibility against solvent vapor above melting point of PEG. By crosslinking of the composite from PEG-grafted carbon black with triisocyanate, the responsibility against solvent vapor disappeared completely. These results indicated that the crystalline structure of PEG plays an important role on the responsibility of electric resistance of PEG-grafted carbon blacks against humidity and alcohol vapor.

KEY WORDS Carbon Black / Poly(ethylene glycol) / Surface Grafting / Composite / Electric Resistance Humidity Sensor / Alcohol Sensor /
\end{abstract}

We have reported the grafting of various vinyl polymers onto carbon black surface by the polymerization of vinyl monomers initiated by initiating groups introduced onto the surface. ${ }^{1-3}$ The polymer-grafted carbon blacks thus obtained were easily and uniformly dispersed in polymer matrices. ${ }^{1-3}$

It is well known that a carbon black-polymer composite prepared from vinyl polymer-grafted carbon black, which was crosslinked with a variety of crosslinking agents, shows a large positive temperature coefficient of electric resistance, i.e., PTC, at near the glass transition temperature of the matrix polymer. ${ }^{4}$ Recently, we have achieved the grafting of crystalline polymers, such as poly(ethyleneimine) (PEI) and poly(ethylene glycol) (PEG), onto carbon black surface by the direct condensation of surface carboxyl groups with terminal amino and hydroxyl groups of these polymers using $N, N^{\prime}$-dicyclohexylcarbodiimide (DCC) as a condensing agent. ${ }^{5}$ Furthermore, we pointed out that the electric resistance of the composite prepared from PEG and PEG-grafted carbon black drastically increases about $10^{4}-10^{5}$ times of initial resistance at the melting point of crystalline polymer. ${ }^{6}$ A plane heater made from the composite, produces uniform heat approximate $60^{\circ} \mathrm{C}$ without any circuit for controlling temperature. These phenomena may be due to a widening of the gaps between the carbon black particles based on the melting of PEG.

In the preceding paper, we have reported that the electric resistance of crystalline PEI-grafted carbon black drastically increased in humidity and alcohol vapor, which is good solvents of grafted chain, but not in

† To whom all correspondence should be addressed. non-solvent vapor, and returned to initial resistance when it was transferred in dry air. ${ }^{7}$ This may be due to a widening of the gaps between the carbon black particles based on the absorption of vapor of good solvent of matrix polymer.

In the present paper, the effects of molecular weight of PEG and temperature on the responsibility of electric resistance of composite from PEG-grafted carbon black against humidity and alcohol vapor were investigated.

\section{EXPERIMENTAL}

\section{Carbon Black and Materials}

Conductive carbon black used was furnace black, Porousblack, obtained from Asahi Carbon Co., Ltd., Japan. The properties of the carbon black was shown in Table I. The content of carboxyl $(\mathrm{COOH})$ and phenolic hydroxyl $(\mathrm{OH})$ groups was determined by use of sodium bicarbonate $^{8}$ and 2,2-diphenyl-1-picrylhydrazyl, ${ }^{9}$ respectively. The carbon black was dried in vacuo at $110^{\circ} \mathrm{C}$ for $48 \mathrm{~h}$ before use.

PEGs used were obtained from Aldrich Chemical Co. and dried in vacuo at $60^{\circ} \mathrm{C}$. The molecular weight and melting point of PEG were summarized in Table II.

4,4'-Azobis(4-cyanopentanoic acid) (ACPA) obtained from Wako Pure Chemical Ind., Ltd., Japan was recrystallized from methanol. DCC obtained from Wako Pure Chemical Ind., Ltd., was dried in vacuo at room temperature. Triisocyanate uses was carbamate of trimethylolpropane with tolylene diisocyanate obtained from Aldrich Chemical Co. Poly(vinyl chloride) film used was commercially available wrapping film whose thickness was about $10-15 \mu \mathrm{m}$. Tetrahydrofuran and other 
Table I. Properties of carbon black used

\begin{tabular}{|c|c|c|c|c|}
\hline \multirow{2}{*}{ Porousblack } & Surface area & Particle size & $\mathrm{OH}$ group & $\mathrm{COOH}$ group \\
\hline & $\mathrm{m}^{2} \mathrm{~g}^{-1}$ & $\mathrm{~nm}$ & $\mathrm{mmolg}^{-1}$ & $\mathrm{mmolg}^{-1}$ \\
\hline Untreated & 447 & 41 & 0.03 & 0 \\
\hline ACPA-treated & 447 & 41 & 0.03 & 0.08 \\
\hline
\end{tabular}

Table II. Grafting of PEG onto carbon black surface

\begin{tabular}{|c|c|c|c|c|c|}
\hline \multirow{2}{*}{ PEG } & \multirow{2}{*}{$M_{n} \times 10^{-3}$} & $\mathrm{mp}$ & \multirow{2}{*}{$\frac{\mathrm{DCC}}{\mathrm{mg}}$} & \multirow{2}{*}{$\begin{array}{c}\text { Grafting } \\
\%\end{array}$} & \multirow{2}{*}{$\frac{\text { Polymer grafted }}{\mu \mathrm{mol} \mathrm{g}^{-1}}$} \\
\hline & & ${ }^{\circ} \mathrm{C}$ & & & \\
\hline PEG-600 & 0.6 & $19-22$ & 25 & 7.2 & 120 \\
\hline PEG-1000 & 1.0 & $36-39$ & 25 & 8.5 & 85.0 \\
\hline PEG-1540 & 1.5 & $44-48$ & 25 & 11.8 & 76.6 \\
\hline PEG-3000 & 3.0 & $55-59$ & 25 & 16.1 & 53.6 \\
\hline PEG-20000 & 10.0 & $58-63$ & 25 & 19.8 & 9.9 \\
\hline
\end{tabular}

Carbon black, $0.25 \mathrm{~g}$; PEG, $1.0 \mathrm{~g}$; THF, $20.0 \mathrm{ml} ; 60^{\circ} \mathrm{C} ; 48 \mathrm{~h}$.

solvents were purified by ordinary methods.

\section{Introduction of Carboxyl Groups onto Carbon Black Surface}

The introduction of carboxyl groups onto carbon black surface was achieved by the treatment with ACPA. ${ }^{10}$ Into a flask, $5.0 \mathrm{~g}$ of carbon black, $0.5 \mathrm{~g}$ of ACPA, and $100 \mathrm{ml}$ of tetrahydrofuran (THF) were charged and the mixture was stirred with a magnetic stirrer under nitrogen at $70^{\circ} \mathrm{C}$ for $48 \mathrm{~h}$. After the reaction, the mixture was centrifuged at $1.5 \times 10^{4} \mathrm{rpm}$ and the supernatant solution was removed by decantation. The resulting carbon black was dispersed in THF and the dispersion was centrifuged again. The procedures were repeated until no more ACPA could be detected in the supernatant solution. The carbon black was dried in vacuo at $45^{\circ} \mathrm{C}$ and stored in a desiccator. The content of carboxyl groups was determined by titration. ${ }^{8}$

\section{Grafting of PEG onto Carbon Black Surface}

The grafting of PEG onto carbon black surface was achieved by the direct condensation of carboxyl groups on the surface with terminal hydroxyl groups of PEG using DCC as a condensing agent. ${ }^{5}$ Into a flask, $0.25 \mathrm{~g}$ of carbon black treated with ACPA, $1.0 \mathrm{~g}$ of PEG, $25 \mathrm{mg}$ of DCC, and $20.0 \mathrm{ml}$ of THF were charged and the reaction mixture was stirred with a magnetic stirrer under nitrogen at $60^{\circ} \mathrm{C}$ for $48 \mathrm{~h}$. After the reaction, the mixture was centrifuged at $1.5 \times 10^{4} \mathrm{rpm}$ and the supernatant solution was removed by decantation. The resulting carbon black was dispersed in methanol and the dispersion was centrifuged again. The procedures were repeated until no more PEG could be detected in the supernatant solution.

The percentage of grafting was determined by the following equation:

$$
\text { Grafting }(\%)=(A / B) \times 100
$$

where $A$ is weight of PEG grafted and $B$ is weight of carbon black charged: the amount of grafted PEG, $A$, was estimated from weight loss when PEG-grafted carbon black was heated at $500^{\circ} \mathrm{C}$ by a thermal analyzer.

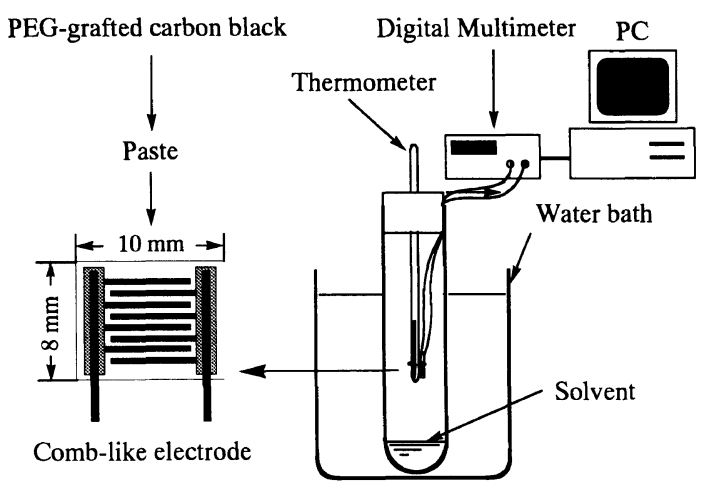

Figure 1. Comb-like electrode and apparatus for the measurement of electric resistance of composite from PEG-grafted carbon black.

\section{Preparation of Composite Resistor from PEG-Grafted Carbon Black}

The preparation of composite resistor from PEG and PEG-grafted carbon black was carried out as follows. Into a test tube, $0.25 \mathrm{~g}$ of PEG-grafted carbon black was dispersed in a small amount (about $1.0-1.5 \mathrm{ml}$ ) of THF and $1.00 \mathrm{~g}$ of PEG was added. The mixture was stirred with a magnetic stirrer at room temperature to produce a composite paste, in which PEG-grafted carbon black was dispersed uniformly in concentrated PEG solution.

\section{Measurement of Electric Resistance}

The measurement of electric resistance of composite from PEG-grafted carbon black was carried out as follows. ${ }^{7}$ The composite paste prepared by the above method was coated onto a comb-like electrode, which was prepared by the screen printing of conductive $\mathrm{Ag} / \mathrm{Pd}$ paste onto a ceramic plate. The thickness of the composite on the electrode was about $120 \mu \mathrm{m}$. The comb-like electrode used in this study was shown in Figure 1.

The electric resistance under humidity and solvent vapor was measured by hanging the composite resistor in a glass container containing water or organic solvent at the bottom. The electric resistance was measured at $25.0^{\circ} \mathrm{C}$ by use of a digital multimeter (Advantest Co., Ltd., Japan: type R6871E-DC). 


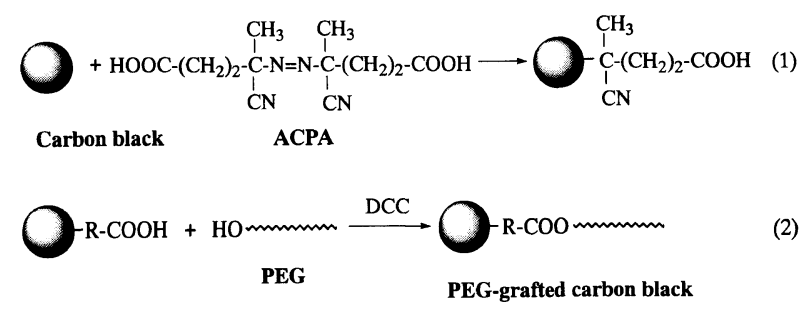

Scheme 1.

\section{RESULTS AND DISCUSSION}

\section{Grafting of PEG onto Carbon Black Surface}

In general, conductive carbon blacks have few functional groups for the grafting sites. Therefore, the introduction of carboxyl groups onto carbon black surface was achieved through the trapping of 4cyanopentanoic acid radicals formed by the decomposition of ACPA by carbon black surface (eq 1). ${ }^{10}$ The carboxyl group content of ACPA-treated carbon black was determined to be $0.08 \mathrm{mmol} \mathrm{g}^{-1}$ by titration as shown in Table I.

The grafting of PEG onto carbon black surface was achieved by the direct condensation of carboxyl groups on the carbon black surface with terminal hydroxyl groups of PEG using DCC as a condensing agent (eq 2). ${ }^{5}$

Table II shows the results of grafting of PEGs having different molecular weight onto carbon black surface. In the absence of DCC, the grafting of these polymers onto carbon black surface scarcely observed. On the contrary, the grafting of PEGs successfully proceeded in the presence of DCC as a condensing agent. The percentage of PEG grafting increased with increasing molecular weight, but the mole number of grafted polymer chain decreased. This may be due to the fact that the steric hindrance of neighboring grafted PEG on the surface enhanced with increasing molecular weight of PEG.

No gelation during the above grafting reaction was observed. This may be due to the fact that a large excess of PEG is reacted with surface carboxyl groups on carbon black. The same tendency was reported in the reaction of functional polymers with surface functional groups on carbon black. ${ }^{5}$

By the grafting of PEG, the carbon black surface changed from hydrophobic to extremely hydrophilic and PEG-grafted carbon blacks readily and uniformly dispersed in solvents, such as methanol, ethanol, and water, and PEG matrix.

\section{Electric Property of PEG-Grafted Carbon Black}

Figures 2 and 3 show the effect of alcohol vapor and humidity on the electric resistance of composite from PEG-1540-grafted carbon black at $25.0^{\circ} \mathrm{C}$, respectively. The electric resistance of the composite drastically increased in methanol, ethanol, and water vapor, which are good solvent for PEG, and immediately returned initial resistance when the composite was transferred in dry air. On the contrary, the electric resistance hardly changed in hexane and acetone vapor, which are nonsolvent of PEG.

Figure 4 shows the effect of methanol vapor on the electric resistance of composite from PEG-1540 and

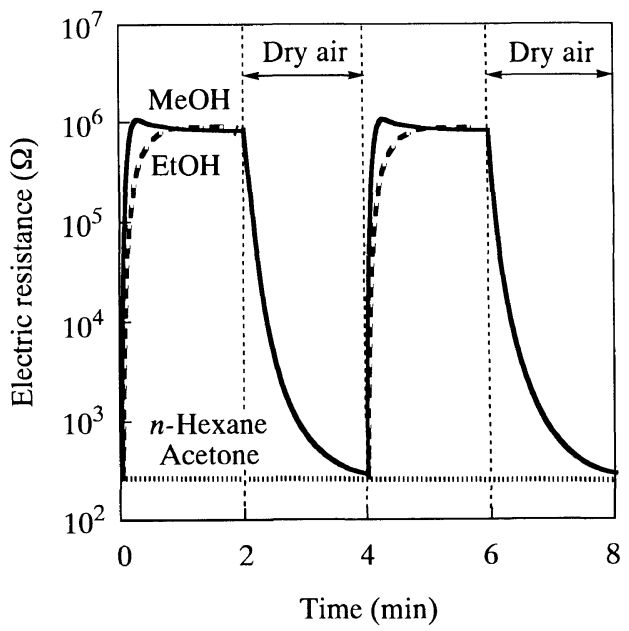

Figure 2. Effect of alcohol vapor on the electric resistance of composite from PEG-1540-grafted carbon black at $25.0^{\circ} \mathrm{C}$.

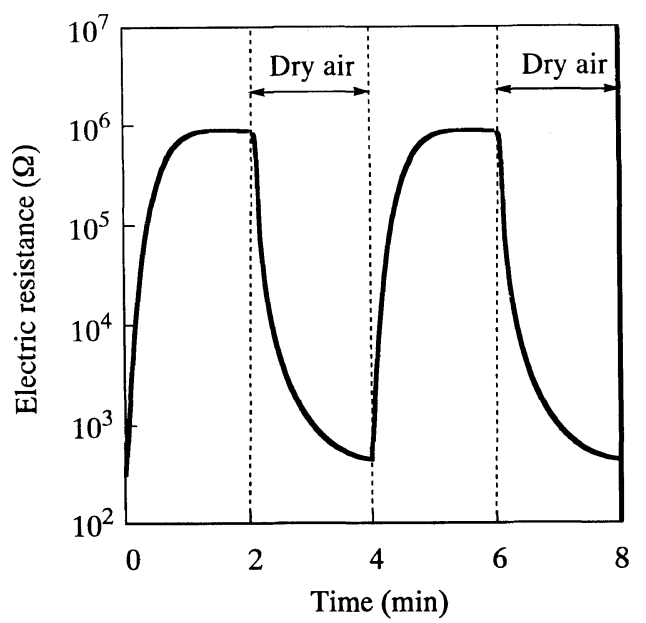

Figure 3. Effect of humidity on the electric resistance of composite from PEG-grafted carbon black at $25.0^{\circ} \mathrm{C}$.

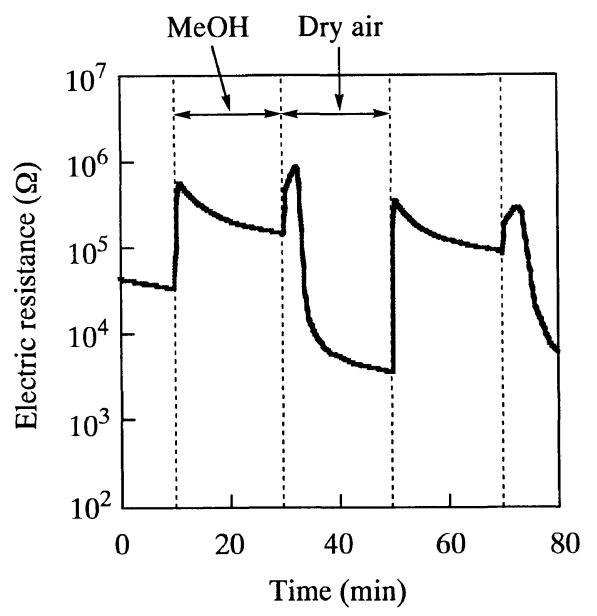

Figure 4. Effect of methanol vapor on the electric resistance of composite from ungrafted carbon black at $25.0^{\circ} \mathrm{C}$.

untreated carbon black. In the case of composite from untreated carbon black, the initial resistance was very large. In addition, the increase of electric resistance in methanol vapor was much smaller than that of PEG-grafted carbon black and hardly returned to initial resistance when it was transferred in dry air as shown in Figure 4. 


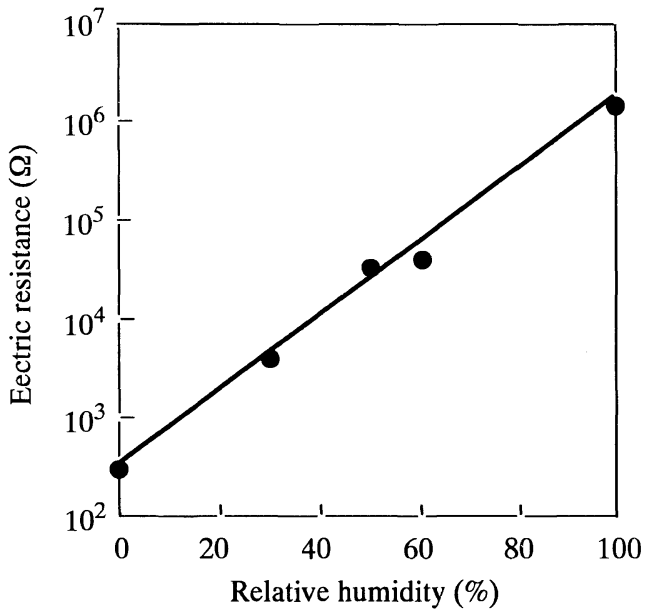

Figure 5. Relationship between electric resistance of composite from PEG-1540-grafted carbon black and relative humidity at $25.0^{\circ} \mathrm{C}$.

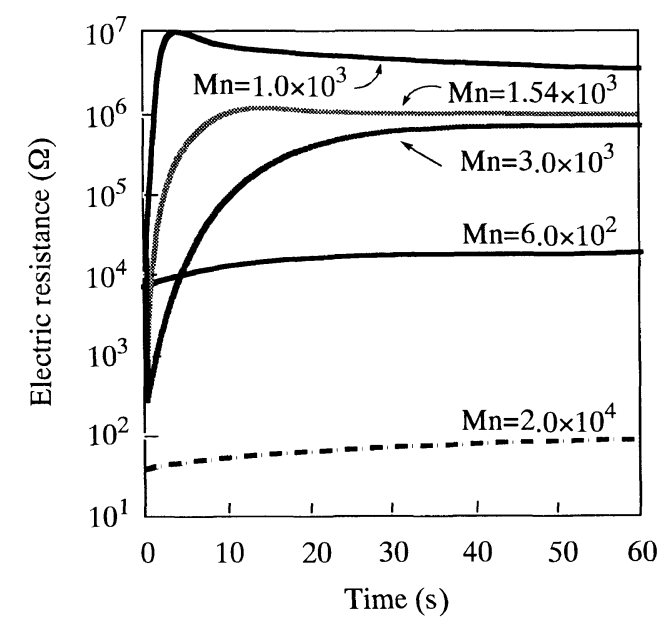

Figure 6. Effect of molecular weight of PEG on the responsibility of electric resistance under methanol vapor at $20.0^{\circ} \mathrm{C}$.

\section{Relationship between Electric Resistance and Relative Humidity}

Figure 5 shows the relationship between electric resistance of composite from PEG-1540-grafted carbon black and relative humidity. It was found that the logarithm of electric resistance is linearly proportional to relative humidity. This indicates that the composite can be applied as a humidity sensor.

Effect of Molecular Weight of PEG on the Responsibility

Figure 6 shows the effect of molecular weight of PEG on the responsibility of electric resistance of composite from PEG-grafted carbon blacks in methanol vapor at $20.0^{\circ} \mathrm{C}$. The sensitivity against methanol vapor was found to decrease with increasing molecular weight of PEG, especially, the electric resistance of PEG-20000-grafted carbon black failed to response against methanol vapor. This suggests that the absorption of methanol into PEG matrix decreases with increasing molecular weight of PEG based on the increase of crystallinity.

In addition, the electric resistance of PEG-600-grafted carbon black also failed to response against methanol vapor. This may be due to the fact that the measuring temperature, $20.0^{\circ} \mathrm{C}$, exceeded the melting point of PEG-600 $\left(19-22^{\circ} \mathrm{C}\right)$, because the electric resistance of

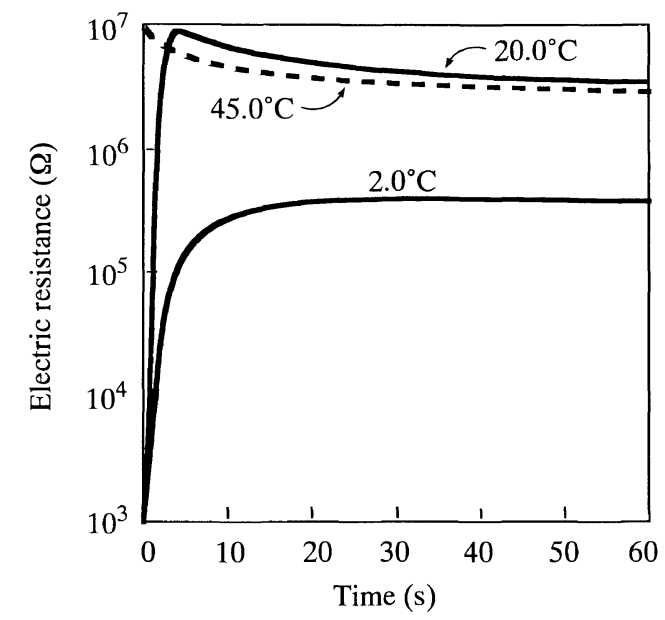

Figure 7. Effect of temperature on the responsibility of electric resistance of composite from PEG-1000-grafted carbon black.

composite from amorphous polymer-grafted carbon black is very high and has no ability to response to solvent vapor. $^{5}$

The initial resistance of the composite decreased depending on the molecule weight of PEG in the

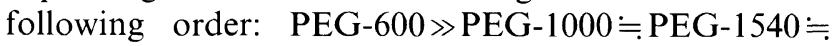
PEG-3000 > PEG-20000. As mentioned above, we have reported the electric resistance of PEG-grafted carbon black shows a large PTC at melting point of PEG, because of widening the gaps between the carbon black particles by melting of PEG. ${ }^{6}$ Therefore, the higher electric resistance of composite from PEG-600-grafted carbon black is considered to be due to PTC. On the other hand, the lower electric resistance of composite from PEG20000-grafted carbon black may be due to shortening of the gaps between the carbon black particles based on the higher crystallinity of PEG-20000.

\section{Effect of Temperature on the Responsibility}

Effect of measuring temperature on the responsibility of composite from PEG-grafted carbon black against methanol vapor was investigated. As shown in Figure 7, PEG-1000-grafted carbon black lost the ability to response to methanol vapor at above melting point of PEG-1000 $\left(45^{\circ} \mathrm{C}\right)$.

On the other hand, PEG-600-grafted carbon black shows the responsibility against methanol vapor at $2.0^{\circ} \mathrm{C}$, below the melting point of PEG-600, in spite of decrease in partial pressure of methanol as shown in Figure 8.

Based on the above results, it was concluded that the crystalline structure of PEG plays an important role on the responsibility of electric resistance of composite from PEG-grafted carbon blacks against methanol vapor.

\section{Effect of Crosslinking of PEG on the Responsibility}

The composite from PEG-grafted carbon black was crosslinked with triisocyanate and the responsibility against methanol vapor was investigated. It became apparent that by crosslinking of PEG matrix, the composite lost the ability to respond against methanol vapor. This may be due to the fact that the absorption of methanol into composite was inhibited by crosslinking. 


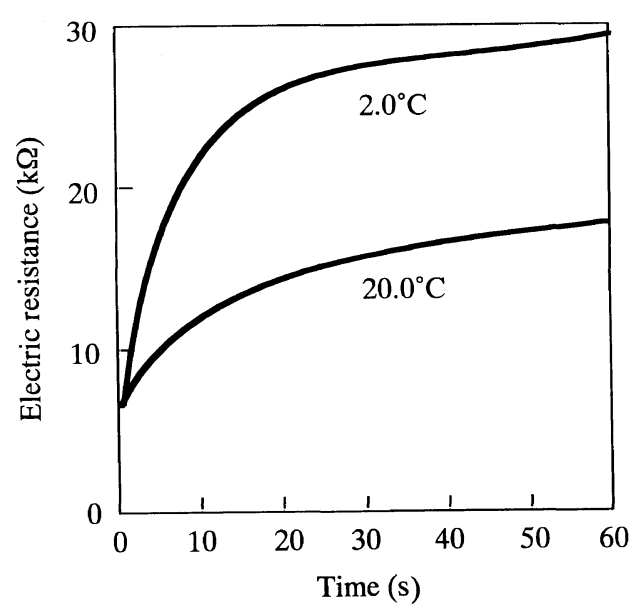

Figure 8. Effect of temperature on the responsibility of electric resistance of composite from PEG-600-grafted carbon black.

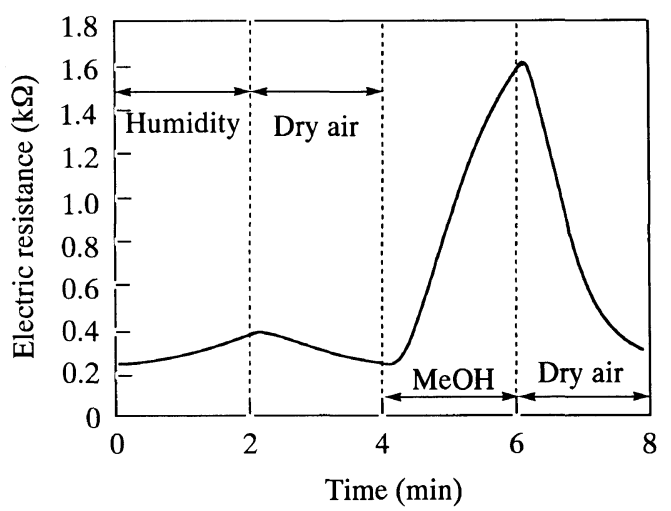

Figure 9. Effect of wrapping of composite from PEG-1540-grafted carbon black with poly(vinyl chloride) film on the responsibility of electric resistance against humidity and methanol vapor at $25.0^{\circ} \mathrm{C}$.

Effect of Wrapping of the Composite with Poly(vinyl chloride) Film on the Responsibility

As mentioned above, the electric resistance of composite from PEG-grafted carbon black has responsibility against humidity and alcohol vapor. Therefore, the comb-like electrode coated with PEG-grafted carbon black was wrapped with poly(vinyl chloride) film (about $10-15 \mu \mathrm{m})$ and selective responsibility of electric resistance against methanol vapor was examined. The results are shown in Figure 9. By wrapping of the element, the responsibility against humidity almost disappeared, but the element retained the responsibility to methanol vapor, although the rate of response delayed. This indicates that by wrapping of the element with poly(vinyl chloride) film, the sensor having selective responsibility against alcohol vapor can be obtained.

\section{CONCLUSIONS}

1. PEG having different molecular weight was successfully grafted onto carbon black surface by direct condensation of terminal hydroxyl groups of PEG with carboxyl groups on the surface using DCC as a condensing agent.

2. The electric resistance of composite from PEGgrafted carbon blacks extremely increased in humidity and alcohol vapor and returned immediately to initial resistance when it was transferred in dry air. This may be due to a widening of the gaps between the carbon black particles based on the absorption of vapor of good solvent of matrix polymer.

3. The sensitivity of electric resistance of the composite decreased with increasing molecular weight of PEG.

4. The electric resistance of the composite from PEG-grafted carbon black lost the responsibility against solvent vapor above melting point of PEG.

5. The crystalline structure of PEG plays an important role on the responsibility of electric resistance of composite from PEG-grafted carbon blacks against good solvent vapor.

Acknowledgment. This study was partly supported by a Grant-in-Aid for Scientific Research (No. 08650795) from the Ministry of Education, Science, Sports and Culture of Japan.

\section{REFERENCES}

1. N. Tsubokawa, Prog. Polym. Sci., 17, 417 (1992).

2. N. Tsubokawa, Kobunshi, 45, 412 (1996).

3. N. Tsubokawa, Shikizai Kyokaishi, 71656 (1998).

4. K. Ohkita and K. Fukushima, Japan Plastics, 3, 6 (1969); ibid., 3, 25 (1969).

5. N. Tsubokawa, M. Hosoya, and J. Kurumada, Reactive Functional Polym., 27, 78 (1995).

6. S. Hayashi, A. Naitoh, S. Machida, M. Okazaki, K. Maruyama, and N. Tsubokawa, Appl. Organometallic Chem., 12, 743 (1998).

7. N. Tsubokawa, S. Yoshikawa, K. Maruyama, T. Ogasawara, and K. Saitoh, Polym. Bull., 39, 217 (1997).

8. D. Rivin, Rubber Chem. Technol., 36, 729 (1963).

9. K. Ohkita and N. Tsubokawa, Carbon, 10, 631 (1972).

10. N. Tsubokawa, K. Magara, and Y. Sone, Nippon Gomu Kyokaishi, 62, 668 (1989). 